\title{
A combined approach of vesicle formulations and microneedle arrays for transcutaneous immunization against hepatitis B virus
}

\author{
Hoang Hirschberg a , Sandra van Kuijk ${ }^{a}$, Jessica Loch ${ }^{\text {a }}$, Wim Jiskoot ${ }^{\text {b }}$, Joke Bouwstra ${ }^{b}$, Gideon Kersten ${ }^{\text {a,* }}$, \\ Jean-Pierre Amorij ${ }^{a}$ \\ ${ }^{a}$ Unit Vaccinology, National Institute for Public Health and the Environment (RIVM), A. van Leeuwenhoeklaan 9, 3721 MA Bilthoven, The Netherlands \\ ${ }^{\mathrm{b}}$ Division of Drug Delivery Technology, Leiden/Amsterdam Center for Drug Research (LACDR), Einsteinweg 55, 2333 CC Leiden, The Netherlands
}

\section{A R T I C L E I N F O}

\section{Article history:}

Received 3 August 2011

Received in revised form 28 January 2012

Accepted 29 January 2012

Available online 4 February 2012

\section{Keywords:}

Microneedles

Elastic vesicles

Transcutaneous immunization

Hepatits B vaccine

\begin{abstract}
A B S T R A C T
In the search for an optimal approach for the transcutaneous immunization (TCI) of hepatitis B surface antigen (HBsAg), two vesicle formulations, L595 vesicles (composed of sucrose-laurate ester and octaoxyethylene-laurate ester) and sPC vesicles (composed of soybean-phosphatidylcholine and Span-80) were prepared and characterized in vitro and in vivo. HBsAg was associated to the vesicles, resulting in SPCHBsAg vesicles $( \pm 170 \mathrm{~nm})$ with $79 \% \mathrm{HBsAg}$ association and L595-HBsAg vesicles $( \pm 75 \mathrm{~nm})$ with only $29 \% \mathrm{HBsAg}$ association.

The vesicles induced in mice via TCI an antibody response only when the skin was pretreated with microneedles. This response was improved by the adjuvant cholera toxin. The sPC-HBsAg vesicle formulations showed to be the most immunogenic for TCI, which was related to the higher degree of $\mathrm{HBsAg}$ association.
\end{abstract}

(ㄷ) 2012 Elsevier B.V. All rights reserved.

\section{Introduction}

Most vaccines are delivered via conventional syringes and needles. Despite their common use, syringes and needles have drawbacks. They can cause fear and pain and in developing countries the spread of diseases due to the reuse of the needles and unsafe injection practice is also an important drawback of conventional needles. To address the limitations of needle injections, a lot of effort is put into needle free delivery systems and routes (Amorij et al., 2010; Bal et al., 2010a; Benson, 2010; Chadwick et al., 2009; Heurtault et al., 2010; Hirschberg et al., 2010; Mann et al., 2009; Prausnitz et al., 2009; Slutter et al., 2010). One very attractive route for vaccination is via the skin. This organ is the largest organ of the body and loaded with immune competent cells. Langerhans cells and dendritic cells in the epidermis and dermis, respectively, play an important role in the capture, uptake and processing of antigens. Before reaching the epidermis and the dermis, the antigen has to pass the outermost layer of the skin, the stratum corneum, which is composed of dead keratinocytes embedded in a lipid matrix. Overcoming this first line of defense is the main challenge in transcutaneous immunization (Bal et al., 2010b; Giudice and Campbell, 2006).

\footnotetext{
* Corresponding author. Tel.: +31 302742925.

E-mail address: gideon.kersten@rivm.nl (G. Kersten).
}

One possible way to overcome the stratum corneum is to associate the antigen with specially designed vesicular systems such as elastic vesicles. Elastic vesicles increase the transport of drugs across the stratum corneum owing to the highly deformable bilayers of these vesicles. The first generation elastic vesicles, transfersomes, were introduced by Cevc and Blume (1992). Transfersomes are composed of phosphatidylcholine and a detergent, such as sodium cholate. Later on, elastic vesicles have also been prepared with other detergents such as sodium deoxycholate, Span 60, Span 65, Span 80, Tween 20, Tween 60 and Tween 80 (El Maghraby et al., 1999, 2000; Oh et al., 2006; Trotta et al., 2004). Most work with elastic vesicles has been done with therapeutic drugs although some studies have been focusing on antigens such as BSA, gap junction protein (Paul et al., 1995, 1998), tetanus toxoid (Gupta et al., 2005) and hepatitis B surface antigen (Mishra et al., 2006). These studies showed, with a same dose of antigen, comparable or significantly higher antibody titers with transcutaneous immunization (TCI) as compared to the conventional intramuscular injection.

In 1998, van den Bergh et al. (1999) introduced a new series of elastic vesicles composed of only surfactants. The bilayer-forming surfactant L595 (sucrose laurate ester) was combined with the micelle forming surfactant PEG-8-L (octaoxyethylene laurate ester). Drug-loaded vesicles showed high transport rates into the stratum corneum (van den Bergh et al., 1999) compared to conventional rigid vesicles making this carrier system promising for antigen delivery. Ding et al. (2010) associated diphtheria toxoid to 
these vesicles and performed immunization studies. They showed with these formulations a significant improvement in the immune response when the skin was pretreated with microneedles (Ding et al., 2010). Microneedles are used to create channels in the skin allowing the passage of antigens through the stratum corneum into the epidermis and the dermis. Ding et al. concluded that the improvement of the immune response was attributed to the use of the microneedles in combination with the use of cholera toxin (CT) as an adjuvant.

The most commonly used adjuvants for TCI are bacterial ADPribosylating exotoxins, such as CT and LT (Glenn et al., 1998). Although exact mechanisms of action have not been completely elucidated, recent observations have shed some light on the mechanism behind the potent adjuvanticity of these toxins. When such adjuvants are added at the site of antigen administration, they provide Langerhans cells with an activation signal to mature and become potent antigen presenting cells (Belyakov et al., 2004). The presence of these adjuvants elicit much higher antibody levels than when antigens are delivered alone (Guebre-Xabier et al., 2003).

In the present study we investigated different TCI strategies for transcutaneous vaccination against hepatitis $B$ surface antigen (HBsAg). HBsAg has been used as such, or associated to different types of vesicles. The formulations were characterized in vitro and evaluated by TCI of mice. Moreover, the enhancement of the immune response against HBsAg after TCI by the use of microneedle arrays, the addition of cholera toxin as adjuvant and the effect of the level of HBsAg-vesicle association were evaluated.

\section{Materials and methods}

\subsection{Materials}

Hepatitis B surface antigen (HBsAg, $1.4 \mathrm{mg} / \mathrm{ml}$ ) was purchased from Serum Institute India. Soybean phosphatidylcholine (Lipoid S100) was provided by Lipoid GmbH (Ludwigshafen, Germany). Span 80 and cholera toxin were purchased from Sigma-Aldrich (Zwijndrecht, The Netherlands). Sucrose-laurate ester (L-595; 30\% mono-, 40\% di-, and 30\% triesters, mean MW 734) was kindly supplied by Mitsubishi Kaei (Tokyo, Japan), octaoxyethylene-laurate ester (PEG-8-L; mean MW 552) was a gift from Lipo Chemicals (Paterson, New Jersey, US) and sodium bistridecyl sulfo succinate (TR70; mean MW 585) was a gift from Cytec B.V (Rotterdam, The Netherlands).

\subsection{Vesicle preparation}

Two kinds of vesicles were prepared: vesicles containing the synthetic surfactants L-595, PEG-8-L and TR70, further called L595 vesicles, and vesicles containing soybean phosphatidylcholine and Span 80, further called SPC vesicles. Both kinds of vesicles were prepared without (empty vesicles) and with $\mathrm{HBsAg}$ associated to the vesicles (L595-HBsAg and sPC-HBsAg). Empty sPC vesicles were also mixed with HBsAg (sPC-HBsAg-mixed).

L595 vesicles consist of the bilayer-forming surfactant L-595, the micelle-forming surfactant PEG-8-L, and the stabilizing surfactant TR70 in a molar ratio of 5:5:1 resulting in a final vesicle concentration of $4 \% \mathrm{w} / \mathrm{w}$. Vesicles were prepared as described by Ding et al. (2010). In brief, the surfactants L-595 and PEG-8-L were dissolved in ethanol and TR70 was dissolved in isopropanol. The solutions were mixed in the proper ratio and the organic solvent was subsequently evaporated overnight in an evaporator (Pierce Reac-

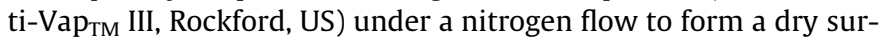
factant film. For the empty vesicles, the surfactant film was dispersed in a $0.01 \mathrm{M}$ phosphate buffer ( $\mathrm{pH}$ 6.5). For the HBsAg associated vesicles (L595-HBsAg), HBsAg diluted to $285 \mu \mathrm{g} / \mathrm{ml}$ in a $0.01 \mathrm{M}$ sodium phosphate $\mathrm{pH}$ (6.5), was used to disperse the remaining surfactant film. The dispersion was sonicated $3 \times 5 \mathrm{~s}$ with a Branson 250 (Branson Ultrasonics, Danbury, UK) using a $1 / 8$ in. microtip. The obtained vesicles were extruded 13 times through a $100 \mathrm{~nm}$ polycarbonate filter (Avanti Polar Lipids, Alabama, US).

S-PC vesicles were prepared as described by Cevc et al. (1998) with slight modifications. In short, soybean-phosphatidylcholine and the surfactant Span 80 , in a ratio (w/w) of 5.7:1, were dissolved in an ethanol-chloroform mixture $(1: 2 \mathrm{v} / \mathrm{v})$. The organic solvent was evaporated overnight in an evaporator (Pierce Reacti-Vap ${ }_{\mathrm{TM}}$ III, Rockford, US) under a nitrogen flow. For empty vesicles, the ob-

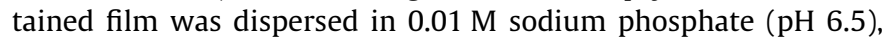
resulting in a final vesicle concentration of $1 \% \mathrm{w} / \mathrm{v}$. For HBsAg associated vesicles (sPC-HBsAg), HBsAg $(285 \mu \mathrm{g} / \mathrm{ml})$ was diluted in $0.01 \mathrm{M}$ sodium phosphate ( $\mathrm{pH}$ 6.5) and $10 \mathrm{ml}$ was used to disperse the film. After $1 \mathrm{~h}$ vortexing (ELMI Intelli-mixer), the dispersion was incubated at room temperature for $2 \mathrm{~h}$ and subsequently sonicated for $15 \mathrm{~s}$ by using a Branson sonifier 250 (Branson Ultrasonics, Danbury, UK), equipped with a $1 / 8$ in. microtip. Finally the sonificated dispersion was extruded (Avestin Inc., Mannheim, Germany) 13 times through a $100 \mathrm{~nm}$ polycarbonate filter (Avanti Polar Lipids, Alabama, US).

For the sPC-HBsAg-mixed formulations, empty sPC vesicles were prepared as described above and HBsAg was added subsequently to a final concentration of $285 \mu \mathrm{g} / \mathrm{ml}$. The sPC-HBsAgmixed formulations were used within $24 \mathrm{~h}$ after preparation.

\subsection{Vesicle characterization}

\subsubsection{Particle size and zeta potential measurements}

The Z-average size and polydispersity index (PDI) of 3 batches of vesicles were measured by dynamic light scattering (DLS) using a Malvern Zetasizer Nano ZS (Malvern, United Kingdom). All measurements were performed at $25^{\circ} \mathrm{C}$ in triplicate.

The zeta potential of three batches of vesicles was measured by laser Doppler electrophoresis using the same device. Each sample was measured three times in a folded capillary cell (Malvern, United Kingdom).

\subsubsection{Size exclusion chromatography (SEC)}

To determine the amount of HBsAg associated with the vesicles, SEC was performed using a Sepharose 6 Fast Flow column of $80 \mathrm{~cm}$ long and with a diameter of $1.6 \mathrm{~cm}$. The column was connected to an AKTA purifier (GE Healthcare, Germany). This automated system was driven by the Windows software package UNICORN 5.11. The column was equilibrated with $0.01 \mathrm{M}$ sodium phosphate ( $\mathrm{pH}$ 6.5). During elution the absorbance of the eluting volume was measured at $215 \mathrm{~nm}$ and fractions of $8 \mathrm{ml}$ were collected. HBsAg and empty vesicles were first characterized by SEC to determine the elution volume of their respective peak fractions in order to characterize the HBsAg associated vesicles. The amount of associated- and free hepatitis B surface antigen in the HBsAg containing vesicle formulations were determined by collection of the peak fractions and subsequent measurement of these fraction in a hepatitis B antigen ELISA and a Lowry protein assay (Lowry et al., 1951).

\subsubsection{Hepatitis B surface antigen ELISA}

For HBsAg quantification a commercial kit of Abbott, Murex HBsAg version 3, was used according to the manufacturer's instructions. In short, the sample was pre-incubated in microwells ( 3 or 4 dilutions) pre-coated with a mixture of mouse monoclonals specific for different epitopes on the 'a' determinant of HBsAg. Affinity purified goat antibody to HBsAg conjugated to horseradish peroxidase was then added. After washing, to remove sample and 
unbound conjugate, peroxidase activity was detected by addition of 3,3',5,5'-tetramethylbenzidine (TMB) substrate and hydrogen peroxide. The enzyme reaction was terminated with $100 \mu \mathrm{l}$ sulfuric acid. Substrate conversion was determined spectrophotometrically at $450 \mathrm{~nm}$. Each ELISA plate contained a reference HBsAg sample for calculation of the antigen concentration in the samples.

\subsubsection{Elasticity measurements}

The elasticity was evaluated by extrusion, as described by Ding et al. (2010) with slight modifications. A fixed volume $(0.5 \mathrm{ml})$ of the sample was pushed through a $30-\mathrm{nm}$ polycarbonate filter by using an extruder (Avanti Polar Lipids Inc., Delftzijl, The Netherlands) at a constant pressure of 1 bar. Every minute, for a time period of $15 \mathrm{~min}$, the volume of vesicle formulation that was pushed through the filter was measured. This volume was plotted in a graph against the time.

Three freshly prepared samples were measured in duplo.

\subsubsection{Immunizations}

Female BALB/c mice (BALB/cOlaHsd), weight $18-20 \mathrm{~g}$ at the start of the experiment were purchased from Harlan (Horst, The Netherlands), and maintained under standardized conditions in the animal facility of the NVI (Netherlands Vaccine Institute, Bilthoven, The Netherlands). All studies have been approved by the Animal Ethical Committee of the RIVM.

Mice of the transcutaneous immunization groups were immunized topically on either intact skin or microneedle pretreated skin. The immunizations were performed as described by Ding et al. (2010). In short, the abdominal skin of the mice was shaved one day before immunization. Just before immunization, mice were anesthesized by intraperitoneal injection of $150 \mathrm{mg} / \mathrm{kg}$ ketamine and $10 \mathrm{mg} / \mathrm{kg}$ xylazine. The shaved skins of the TCI groups, were first wiped with $70 \%$ ethanol and left to dry. The skin was then pierced using a $4 \times 4$ microneedle array of $300 \mu \mathrm{m}$ needles. A metal ring was placed over the pierced area and $70 \mu \mathrm{l}$ of the formulation was spread equally over this fixed area of $2 \mathrm{~cm}^{2}$. For the TCI groups without a microneedle pretreatment, a same metal ring was placed on dry shaved with ethanol wiped skin and $70 \mu \mathrm{l}$ of the formulation was spread equally over the fixed area. A metal lid was placed on top of the metal ring for occlusive application. After $1 \mathrm{~h}$ application, the mice were washed under the tap with lukewarm water and placed back in their cages. The HBsAg dose used for transcutaneous immunization was $20 \mu \mathrm{g}$. When cholera toxin was used, $100 \mu \mathrm{g}$ of the toxin was added to the formulation prior to application.

In all in vivo experiments a control group was included receiving an intramuscular injection $(500 \mu \mathrm{l})$ with hepatitis B surface antigen $(2 \mu \mathrm{g})$ adjuvanted with $144 \mu \mathrm{g}$ aluminum hydroxide. Two weeks after primary immunization, blood was collected prior to a booster vaccination by an orbitapunction under isofluran $/ \mathrm{O}_{2}$ anesthesia. Four weeks after the booster vaccination, all mice were sacrificed by bleeding under isofluran $/ \mathrm{O}_{2}$ anesthesia.

\subsubsection{Serum antibody levels measured by ELISA}

Polystyrene 96-well microtiter plates were coated with $0.2 \mu \mathrm{g} /$ well HBsAg diluted in $8 \mathrm{mM}$ phosphate buffered saline (PBS), $\mathrm{pH}$ 7.2 (Gibco, Paisly, UK). The plates were incubated overnight at room temperature. The plates were washed with tap water containing $0.1 \%(\mathrm{w} / \mathrm{v})$ Tween 80 . Serum was added in series of three fold dilutions. All dilutions of serum were prepared in PBS containing $0.5 \%(\mathrm{w} / \mathrm{v})$ BSA and $0.05 \%(\mathrm{w} / \mathrm{v})$ Tween 80 . After $2 \mathrm{~h}$ incubation at $37^{\circ} \mathrm{C}$ the plates were washed and goat anti mouse immunoglobulin conjugated to horse radish peroxidase (IgG-HRP, IgG1-HRP or IgG2a-HRP) from Southern Biotech, Birmingham, Ala, USA) was added in the concentration that was given by the manufacturer $(1 / 5000)$ and the plates were incubated for $1.5 \mathrm{~h}$ at $37^{\circ} \mathrm{C}$. After washing the plates, $100 \mu \mathrm{l}$ TMB substrate solution (see above) was added to each well. After $10 \mathrm{~min} 50 \mu \mathrm{l} 2 \mathrm{M} \mathrm{H}_{2} \mathrm{SO}_{4}$ was added to each well and absorbance measured at $450 \mathrm{~nm}$. The titer was determined as the dilution factor at which the absorbance was $50 \%$ of maximum absorbance (plateau value).

\subsubsection{Statistics}

Antibody titers are expressed as the mean $\log _{10}$ titer of the responding mice plus the standard errors of the means. Statistical evaluations are done with a Student $t$-test (one way ANOVA) by using a two tailed distribution and a two sample unequal variance.

\section{Results}

\subsection{Characterization}

\subsubsection{Size and zeta potential}

Empty vesicles (L595 and SPC) and hepatitis B associated vesicles (L595-HBsAg and sPC-HBsAg) were prepared. Empty sPC vesicles have also been mixed with HBsAg (sPC-HBsAg-mix). The vesicle size and the zeta potential of all formulations are shown in Table 1.

The SPC vesicles were larger than the $\mathrm{L} 595$ surfactant vesicles and showed a less negative zeta potential (see Table 1). For both kinds of vesicles, the association of HBsAg with the vesicles resulted in a decrease of the (negative) zeta potential and no significant changes in the vesicle sizes. For all formulations the polydispersity index (data not shown) was smaller than 0.3 , on a scale from 0 to 1 , and only one size population was detected.

In addition, SPC-HBsAg and sPC-HBsAg-mix formulations analyzed by DLS showed a similar size and zeta potential.

\subsubsection{HBsAg incorporation}

Free and associated HBsAg in the vesicle based formulations were separated by SEC (Fig. 1) and the HBsAg content in the peak fractions was determined by ELISA. Empty vesicles showed a main peak eluting at about $60 \mathrm{ml}$, whereas HBsAg showed a main peak at about $80 \mathrm{ml}$ (Fig. 1A). As determined by ELISA, 22\% of the applied HBsAg was recovered in the $60 \mathrm{ml}$ peak and $62 \%$ in the $80 \mathrm{ml}$ peak (see Fig. 1A), resulting in a total recovery of $84 \%$. With DLS a Zaverage of $\sim 30 \pm 3 \mathrm{~nm}$ was measured in the $80 \mathrm{ml}$ fraction and of $120 \pm 10 \mathrm{~nm}$ in the $60 \mathrm{ml}$ fraction. This indicates the presence of HBsAg aggregates, eluting in the $60 \mathrm{ml}$ fraction.

L595-HBsAg vesicles showed 2 peaks (Fig. 1B), one corresponding to the vesicle peak $(60 \mathrm{ml})$ and one to the HBsAg peak $(80 \mathrm{ml})$. ELISA showed that the vesicle and HBsAg peak contained $29 \%$ and $50 \%$ of the formulated HBsAg, respectively.

sPC-HBsAg eluted in one peak (Fig. 1C), corresponding to the vesicle peak $(60 \mathrm{ml})$ containing $79 \% \mathrm{HBsAg}$. In contrast, empty sPC vesicles mixed with $\mathrm{HBsAg}$, SPC-HBsAg-mix, eluted in two peaks with 7\% $\mathrm{HBsAg}$ recovery in the $60 \mathrm{ml}$ peak and 75\% $\mathrm{HBsAg}$ recovery in the $80 \mathrm{ml}$ peak (Fig. 1D).

In summary, the results of SEC and ELISA of the SEC-fractions showed different $\mathrm{HBsAg}$ association for the different $\mathrm{HBsAg}$

Table 1

Size and zeta potential (mean \pm SD; $n=3$ ) of L595 vesicles and SPC vesicles, with and without hepatitis B surface antigen (associated or mixed).

\begin{tabular}{lcc}
\hline Formulation & Z-average size $(\mathrm{nm})$ & Zeta potential $(\mathrm{mV})$ \\
\hline L595 & $75.1 \pm 7.6$ & $-57.1 \pm 1.4$ \\
L595-HBsAg & $68.6 \pm 9.7$ & $-36.6 \pm 4.4$ \\
SPC & $170.0 \pm 22.2$ & $-17.9 \pm 3.4$ \\
SPC-HBsAg & $150.4 \pm 12.9$ & $-10.2 \pm 2.9$ \\
SPC-HBsAg-mix & $153.0 \pm 2.7$ & $-7.5 \pm 1.7$ \\
HBSAg & $29.2 \pm 1.9$ & $-14.1 \pm 2.9$ \\
\hline
\end{tabular}



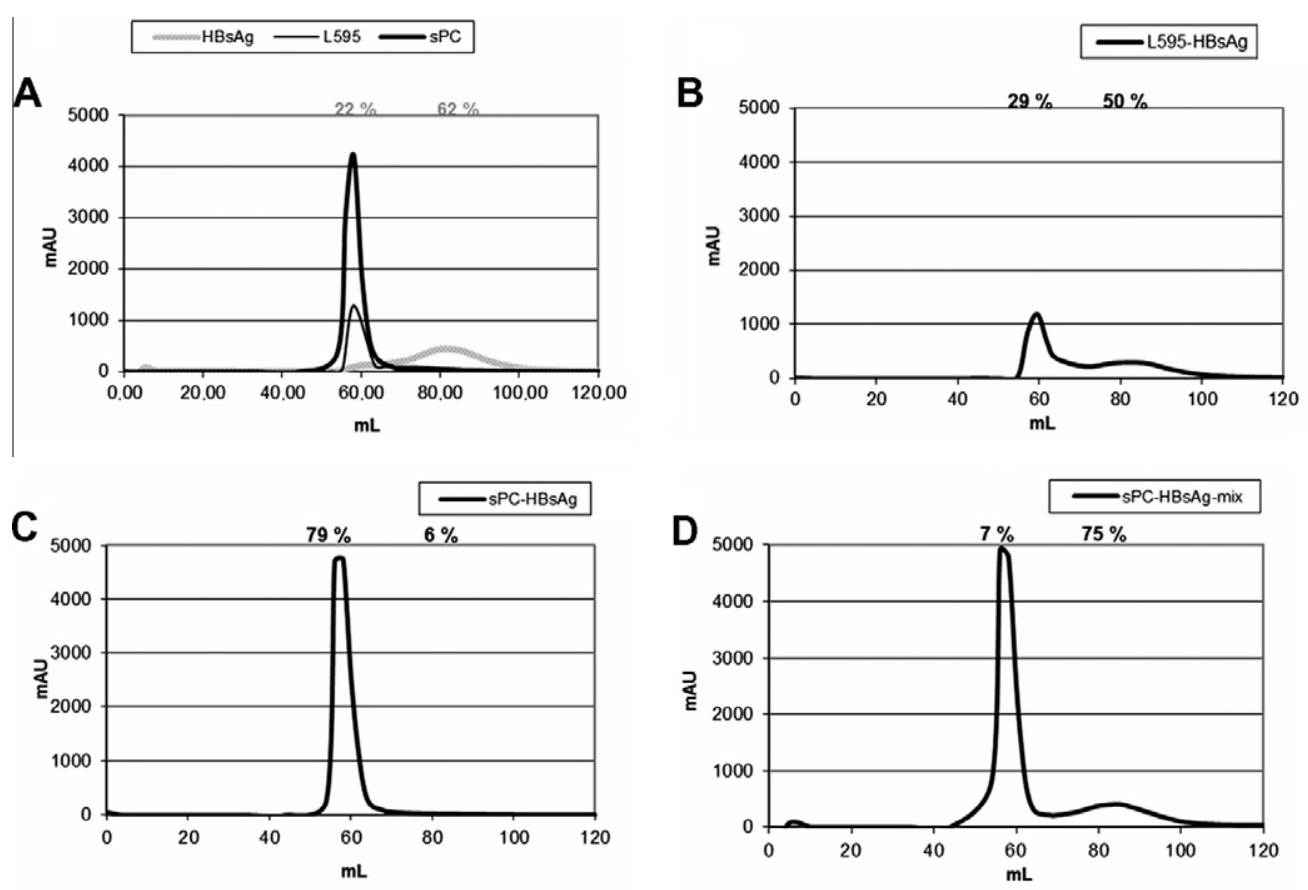

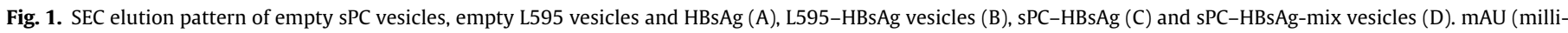

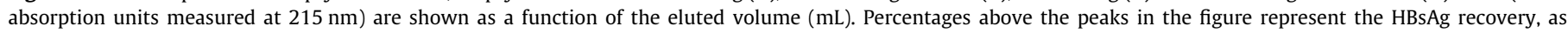
measured with an ELISA, in the $60 \mathrm{ml}$ and $80 \mathrm{ml}$ peak fractions of the HBsAg elution pattern (A) and of the vesicle elution patterns (B-D).

formulations. L595 vesicles had a low HBsAg association and a considerable amount of free HBsAg. In contrast sPC-HBsAg vesicles had a high HBsAg association and a low amount of free HBsAg. Moreover, mixing of empty sPC vesicles with $\mathrm{HBsAg}$ resulted in very low association of HBsAg, even lower than that shown for the HBsAg-L595 vesicles. In the "vesicle peak" $(60 \mathrm{ml})$ of the sPC-HBsAg-mix (1D), an even lower recovery of HBsAg was found as compared to HBsAg (Fig. 1A).

\subsubsection{Elasticity of vesicles}

The elasticity of the vesicles was measured by extrusion through a $30 \mathrm{~nm}$ filter (as shown in Fig. 2). Fig. 2 shows that empty SPC- and L595 vesicle formulations and plain HBsAg passed within $10 \mathrm{~min}$ through the $30 \mathrm{~nm}$ filter. The sPC-HBsAg-mix formulation too, was extruded within $10 \mathrm{~min}$. On the contrary, when $\mathrm{HBsAg}$

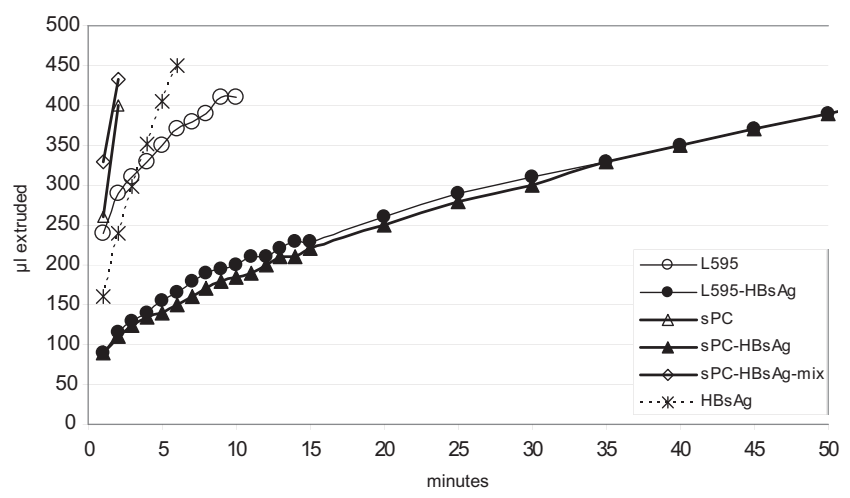

Fig. 2. Elasticity measurements of L595- and sPC-Sp80 vesicles. A fixed volume of $500 \mu \mathrm{l}$ formulation was extruded under a constant pressure of 1 bar through a $30 \mathrm{~nm}$ filter. The volume extruded was measured in time. At least three samples were freshly prepared and measured in duplicate (standard deviation of extruded volume of all measurements $<10 \%$ ). For clarity reasons, results of only one measurement are shown. was associated to the vesicles, (sPC-HBsAg and L595-HBsAg vesicles) more than 50 min were needed to pass through the filter. Apparently the association of HBsAg to the vesicles decreased the extrusion speed.

DLS measurements of the samples before and after extrusion revealed an increase in size of all samples containing HBsAg after extrusion. For L595-HBsAg, sPC-HBsAg and sPC-HBsAg-mix, an increase in size of respectively $87 \%, 37 \%$ and $60 \%$ was found after extrusion. This increase in size can most probably be explained by the aggregation of HBsAg after extrusion. With the HBsAg sample, an increase in size was also measured (from 30 to $150 \mathrm{~nm}$ ). Fig. 2 shows that the aggregates of the HBsAg sample do not block the filter. Therefore, the slower extrusion speed of the HBsAg associated vesicles was not attributed to the obstruction of the filter caused by aggregation of HBsAg.

Empty vesicles in contrary showed the same size before and after extrusion. These vesicles can be considered as elastic.

\subsection{Immunization}

\subsubsection{TCI with HBsAg}

Before we investigated the use of vesicle formulations for TCI, an in vivo study with plain HBsAg was performed to evaluate whether new formulations are needed for TCI. In this in vivo experiment, HBsAg, non-adjuvanted or adjuvanted with cholera toxin (CT), was applied on intact skin or on microneedle pretreated skin. Only when the skin was pretreated with microneedles (see Fig. 3), prior to the occlusive application of the formulations, a HBsAg-specific antibody titer was observed. In the group without adjuvant but with microneedle pretreatment, three animals responded with detectable IgG and IgG1 antibodies. In one animal, IgG2a was detected. In the presence of cholera toxin, significant higher anti-HBsAg antibody responses ( $p=0.0012$ ) were induced as compared to the nonadjuvanted formulations and more responders were observed. The titers were, however, still significantly lower ( $p<0.001$ for IgG and $\operatorname{IgG} 1, p=0.036$ for IgG2a) than those induced after intramuscular 


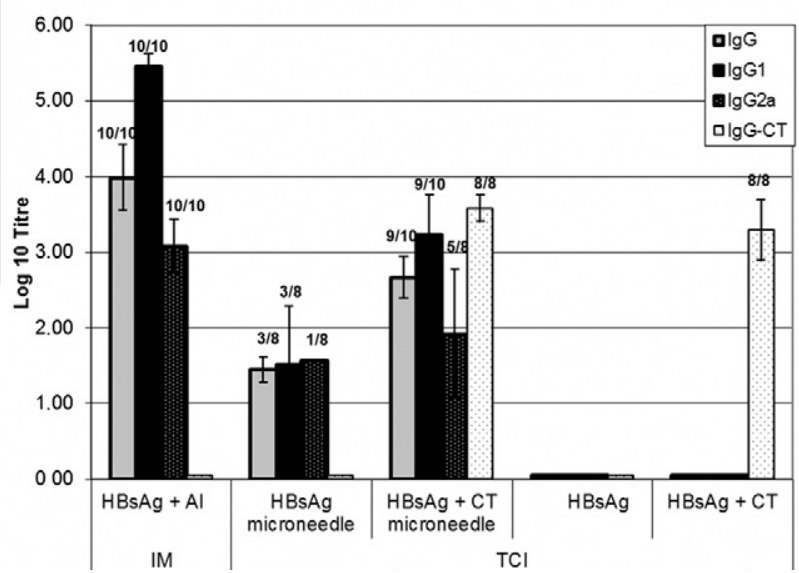

Fig. 3. $\mathrm{TCI}$ with $\mathrm{HBsAg}$ : Antibody titers against $\mathrm{HBsAg}$ and cholera toxin (CT) in mice $(n=10)$ after 45 days (two immunizations). Mice were immunized intramuscularly or transcutaneously. Intramuscular immunization was done with alum adjuvanted HBsAg. The transcutaneous immunization was done by topical dermal application of HBsAg formulations on either intact skin or microneedle pretreated skin. HBsAg was applied transcutaneously in the presence and absence of cholera toxin (CT) as an adjuvant. Numbers above the bars indicate the number of responders. Groups depicted with less than 10 animals were groups in which some animals died as a result of the narrow therapeutic window of the anesthesia used during transcutaneous application.

injection with an alum adjuvanted HBsAg formulation. TCI with the combination of HBsAg and CT did not result in $100 \%$ of the mice responding with detectable HBsAg specific IgG titers.

The serum of the immunized mice was additionally screened for IgG antibodies against CT. Antibody responses against CT were induced in the TCI groups irrespective of the microneedle pretreatment of the skin.

So, TCI of HBsAg resulted in an immune response only when the skin was pretreated with microneedles. This immune response was however still inferior to the response obtained with i.m injection of the conventional alum-adjuvanted vaccine formulation. In order to improve the immune response via $\mathrm{TCI}$, vesicle formulations have been prepared.

\subsubsection{TCI via intact skin with hepatitis B surface antigen associated to vesicles}

sPC-HBsAg formulations and L595-HBsAg formulations were prepared and applied occlusively with and without CT on intact skin in the absence of microneedle pretreatment. The serum was analyzed for IgG titers against HBsAg. As shown in Fig. 4, the vesicle formulations did not induce IgG responses against $\mathrm{HBsAg}$ via TCI on intact skin. Since no response against HBsAg was measured with the TCI groups, the antibody response against CT was also measured. The high IgG responses against CT indicate that an immune response in these mice was possible after TCI.

The absence of an immune response against HBsAg and the strong immune response against CT might be attributed to a higher immunogenicity of $\mathrm{CT}$ and/or a higher permeability of the skin for CT compared to HBsAg. Since TCI on intact skin with the CT adjuvanted vesicle formulations of $\mathrm{HBsAg}$ did not contribute to the immune response against $\mathrm{HBsAg}$, the usefulness of vesicle formulation in combination with microneedles was assessed.

\subsubsection{TCI on microneedle pretreated skin with $\mathrm{HBsAg}$ associated to vesicles}

When CT adjuvanted vesicle formulations were occlusively applied on microneedle pretreated skin, detectable $\operatorname{IgG}$ titers were induced (see Fig. 5). After prime immunization, sPC-HBsAg formulations induced similar IgG titers as compared to those

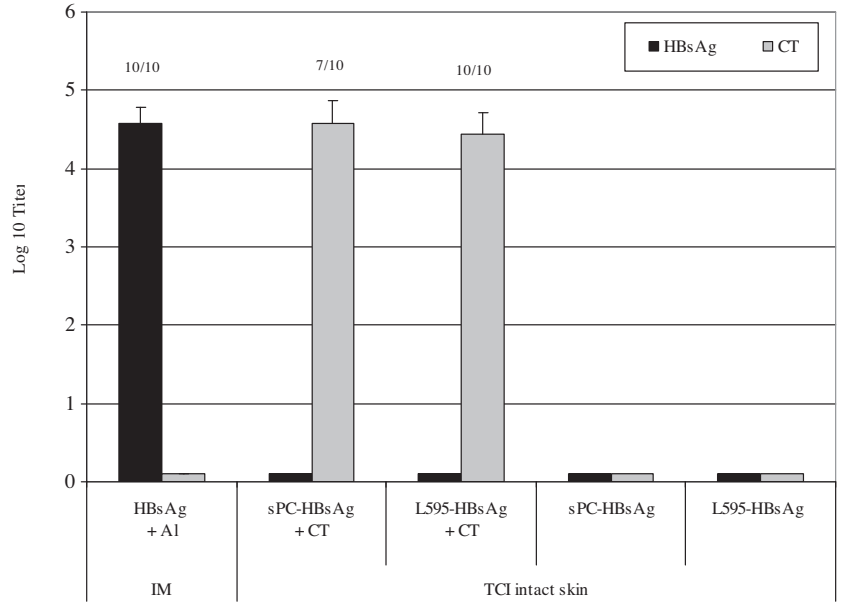

Fig. 4. TCI with HBsAg-vesicle formulations on intact skin: IgG responses against HBsAg and CT after 42 days (two immunization schedule) in mice $(n=10)$. Mice were immunized via intramuscular injection of alum adjuvanted HBsAg or via TCI by the occlusive application of vesicle formulations on intact skin. Soybean phosphatidylcholine vesicles (sPC-HBsAg) and detergent (L595-HBsAg) vesicles have been used. The numbers above the bars indicate the number of responders out of the numbers of animals in a group. Al: aluminum hydroxide; $\mathrm{CT}$ : cholera toxin; IM: intramuscular.

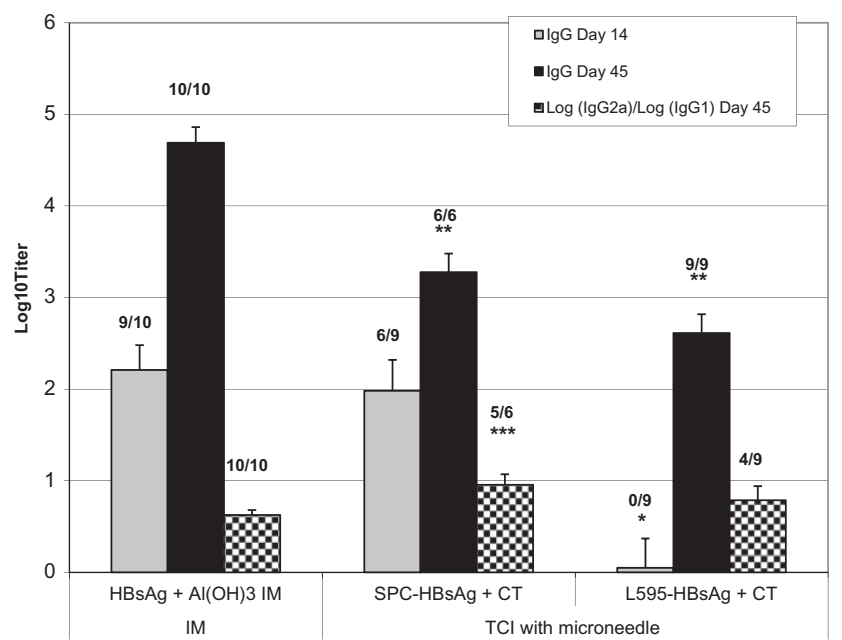

Fig. 5. TCI with HBsAg-vesicle formulations on microneedle pretreated skin: The IgG response in mice was measured after 14 days (one immunization) and 45 days (two immunizations). IgG2a/IgG1 ratios were only measured after 45 days. Mice received either an intramuscular injection or a microneedle pretreatment of the skin followed by the occlusive application of vesicle formulations. Soybean phosphatidylcholine vesicles (sPC-HBsAg) and detergent (L595-HBsAg) vesicles have been used. $(*)$ indicate IgG responses after 14 days that were significantly $(p<0.05)$ lower than the IgG response after intramuscular injection at day 14. $(* *)$ indicate IgG responses after 45 days that were significant $(p<0.05)$ lower than the IgG response after intramuscular injection at day 45. (***) indicate IgG2a/IgG1 ratios that were significant $(p<0.05)$ higher than the $\operatorname{IgG} 2 \mathrm{a} / \operatorname{IgG} 1$ ratio after intramuscular injection. The numbers above the bars indicate the number of responders out of the numbers of animals in a group.

induced by intramuscular injections of alum adjuvanted HBsAg vaccine. In contrary, L595-HBsAg formulations did not yet induce IgG titers after prime immunization. For L595-HBsAg an immune response was only measured after booster immunization. Not only the immune response that was measured after 14 days, but also the higher IgG titers after 45 days indicate the better performance of the SPC-HBsAg vesicles as compared to the L595-HBsAg vesicles. In addition, the highest IgG2a/IgG1 ratio was found with the 
sPC-HBsAg vesicles indicating a more Th1 skewed response as compared to the i.m. injection or the L595-HBsAg vesicles.

Fig. 5 demonstrates the better performance of the sPC-HBsAg vesicles as compared to the L595-HBsAg vesicles. Beside a different vesicle composition, these vesicles also differ in the amount of HBsAg association. The HBsAg association for SPC-HBsAg vesicles was at least $75 \%$ compared to $25 \%$ for the L595-HBsAg vesicles. Since the sPC-HBsAg formulations performed better than the L595-HBsAg vesicles, further research was performed with the sPC-HBsAg vesicles.

In order to determine the effect of the association degree of $\mathrm{HBsAg}$ on the immune response against $\mathrm{HBsAg}$ upon $\mathrm{TCI}$, formulations were prepared with $\mathrm{HBsAg}$ associated to the vesicles (sPCHBsAg) and with empty sPC vesicles mixed with HBsAg (sPCHBsAg-mix). In the first formulation, 75\% of HBsAg was associated to the vesicles whereas in the latter formulation, less than $10 \%$ of HBsAg was associated to the vesicles. The formulations were occlusively applied on microneedle pretreated skin in the absence and presence of cholera toxin.

Fig. 6 shows that TCI with sPC-HBsAg formulations resulted in more responders than the SPC-HBsAg-mix formulations after prime (resp. 3 out of 8 and 0 out of 10) and booster (resp. 5 out of 6 and 6 out of 8 ) vaccination, indicating the superior performance of the sPC-HBsAg formulations over the sPC-HBsAg-mix formulations. When CT was included, a better performance (more responders) after $\mathrm{TCI}$ with $\mathrm{sPC}-\mathrm{HBsAg}$, as compared to $\mathrm{SPC}-\mathrm{HBsAg}-$ mix, was only seen after the prime immunization (respectively 9 / 10 and 1/10). In the presence of $C T$ after boost vaccination, the sPC-HBsAg formulations with higher HBsAg association, did not perform better than the SPC-HBsAg formulations with a lower HBsAg association.

In line with previous experiments (see Fig. 3) TCI with HBsAg formulations without and in combination with $\mathrm{CT}$ resulted in non-responders, even after booster vaccination. The surplus value of the sPC-HBsAg vesicle formulations over the HBsAg formula-

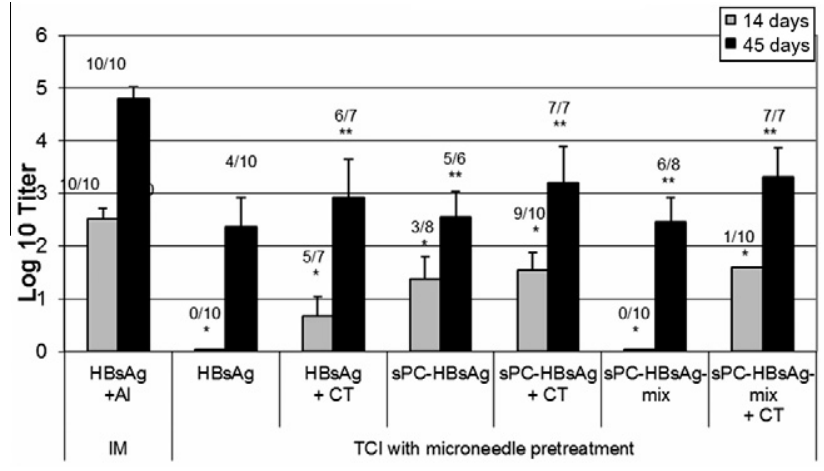

Fig. 6. TCI with $\mathrm{HBsAg}$ and $\mathrm{SPC}-\mathrm{HBsAg}$ formulations, with and without $\mathrm{CT}$, on microneedle pretreated skin: HBsAg antibody responses (IgG) in mice $(n=10)$ after intramuscular, and transcutaneous immunization after microneedle pretreatment. Responses were measured after 14 days (one immunization) and 45 days (two immunizations). HBsAg: hepatitis B surface antigen, $\mathrm{Al}$ : aluminum hydroxide, CT: Cholera toxin, SPC-HBsAg: SPC vesicles with HBsAg associated to the vesicles, SPCHBsAg-mix: empty sPC vesicles mixed with HBsAg. The skin of mice from the transcutaneous groups, was treated with microneedles prior to occlusive dermal application. Numbers above the bars indicate the numbers of responders out of the total number of animals. Groups with less than 10 animals were groups in which animals died during the experiment due to the long anesthesia time. The averages shown in the figure are averages of only the responders. $(*)$ indicate IgG responses after 14 days that were significantly $(p<0.05)$ different from the IgG response after intramuscular injection at day 14. (**) indicate $\operatorname{IgG}$ responses after 45 days that were significant $(p<0.05)$ different from the IgG response after intramuscular injection at day 45 . tions for TCI is expressed in the higher number of responder animals both after prime and booster vaccination.

\section{Discussion}

The objective of the current study was to optimize the TCI approach for vaccination with HBsAg. We investigated approaches based on vaccine application on intact skin or microneedle pretreated skin. Furthermore different types of vesicle formulations as well as the role of HBsAg association level to the vesicles and the immune potentiation by CT were investigated.

Overcoming the skin barrier by microneedle pretreatment was crucial for the induction of HBsAg specific IgG titers which is in line with previous studies with other antigens (Bal et al., 2010b; Ding et al., 2009). In contrast, microneedle pretreatment was not critical for the induction of IgG antibody response against CT. Both groups, with the skin left intact and with microneedle pretreated skin, showed comparable CT-specific antibody responses. Ding et al. hypothesized that, although the size of CT is above $500 \mathrm{Da}$, a part of the CT might have entered the (epi)dermis via hair follicles resulting in antibody responses against CT (Ding et al., 2009). Apparently, CT is a very potent antigen when applied via the skin. Pre-existing immunity may hamper repeated use of CT adjuvanted vaccines. Although not clear yet, it has to be taken into account when considering $\mathrm{CT}$ as an adjuvant for human vaccines. The low responses and low numbers of HBsAg responders might be a result of several factors, such as a low intrinsic immunogenicity of HBsAg. DC studies in our lab confirm that HBsAg is not able to induce DC maturation at concentrations as high as $100 \mathrm{ug} / \mathrm{ml}$ while CT is able to induce DC maturation at concentrations as low as $2 \mu \mathrm{g} / \mathrm{ml}$. Another explanation, although not measured, is the limited amount of HBsAg reaching the APC's as a result of a limiting application time (only $1 \mathrm{~h}$ ), the antigen concentration (Ding et al., 2008) and/ or the large size of HBsAg (Bal et al., 2010c).

In the search for an optimal approach for TCI with HBsAg, two vesicle formulations, L595 and SPC vesicles, were studied. Both HBsAg vesicle formulations did not result in detectable antibody responses in mice without microneedle pretreatment. For L595 vesicle formulations of diphtheria toxoid, comparable results were shown by Ding et al. They related the absence of an antibody response to the limited elasticity of the L595 vesicle formulations. On the other hand, Mishra et al. (2006) obtained good HBsAg antibody responses with their SPC vesicles. There are several differences between our study and that of Mishra, such as the different dose of HBsAg in the two studies, the way of application and most probably also the preparation method of the vesicle formulation. The latter was not described in detail in the previous study.

Cholera toxin had no adjuvant effect on the HBsAg titers when the formulations were applied on intact skin. On microneedle pretreated skin, CT did show an adjuvant effect when included in HBsAg and HBsAg-vesicle formulations. The best adjuvant effect of CT was shown with the SPC-HBsAg formulations as compared to the HBsAg and the SPC-HBsAg-mix formulations. This might be caused by the co-association of both $\mathrm{CT}$ and HBsAg to the vesicles in the SPC-HBsAg formulations. An adjuvant effect of CT, coadministered with hepatitis $\mathrm{B}$, was also found by Isaka et al. (2001) with an intranasal vaccine. Masheswari et al., 2011 showed a mixed Th1/Th2-like immune response when CT was co-administered with HbsAg encapsulated niosomes. CT is believed to activate Langerhans cells to mature and become potent antigen presenting cells (Belyakov et al., 2004; Glenn et al., 2007) although the exact mechanism of action of $\mathrm{CT}$ is not clear yet.

The potency for hepatitis B vaccines is determined by seroconversion as a function of the dose. In this study the most promising TCI procedure includes the use of SPC-HBsAg vesicles adjuvanted 
with CT and application on microneedle pretreated skin. In mice, this resulted in comparable seroconversion as compared to the conventional i.m. vaccination.

Several other TCI approaches have been investigated for hepatitis B vaccination (Lebre et al., 2011), such as particle mediated epidermal delivery (DNA-coated gold microparticles are used to transfect target tissues), epidermal powder immunization, and vesicular approaches (ethosomes, cationic transfersomes and niosomes). All these approaches resulted in a combined Th1/Th2-like response. Our results also indicate a more balanced Th response, compared to the Th2 biased aluminum hydroxide adjuvated vaccine.

To have this TCI procedure fully optimized, future research should include investigation of seroconversion as function of dose and application time.

\section{Acknowledgements} Pa.6.

\section{References}

Amorij, J.P., Hinrichs, W., Frijlink, H.W., Wilschut, J.C., Huckriede, A., 2010. Needlefree influenza vaccination. Lancet Infect. Dis. 10, 699-711.

Bal, S.M., Ding, Z., Kersten, G.F., Jiskoot, W., Bouwstra, J.A., 2010a. Microneedlebased transcutaneous immunisation in mice with $\mathrm{N}$-trimethyl chitosan adjuvanted diphtheria toxoid formulations. Pharm. Res. 27, 1837-1847.

Bal, S.M., Ding, Z., van Riet, E., Jiskoot, W., Bouwstra, J.A., 2010b. Advances in transcutaneous vaccine delivery: do all ways lead to Rome? J. Control Release $148,266-282$.

Bal, S.M., Slutter, B., van Riet, E., Kruithof, A.C., Ding, Z., Kersten, G.F., Jiskoot, W. Bouwstra, J.A., 2010c. Efficient induction of immune responses through intradermal vaccination with $\mathrm{N}$-trimethyl chitosan containing antigen formulations. J. Control Release 142, 374-383.

Belyakov, I.M., Hammond, S.A., Ahlers, J.D., Glenn, G.M., Berzofsky, J.A., 2004 Transcutaneous immunization induces mucosal CTLs and protective immunity by migration of primed skin dendritic cells 2 . J. Clin. Invest. 113, 998-1007.

Benson, H.A., 2010. Elastic liposomes for topical and transdermal drug delivery. Methods Mol. Biol. 605, 77-86.

Cevc, G., Blume, G., 1992. Lipid vesicles penetrate into intact skin owing to the transdermal osmotic gradients and hydration force 1 . Biochim. Biophys. Acta $1104,226-232$

Cevc, G., Gebauer, D., Stieber, J., Schatzlein, A., Blume, G., 1998. Ultraflexible vesicles, transfersomes, have an extremely low pore penetration resistance and transport therapeutic amounts of insulin across the intact mammalian skin. Biochim. Biophys. Acta 1368, 201-215.

Chadwick, S., Kriegel, C., Amiji, M., 2009. Delivery strategies to enhance mucosal vaccination. Expert Opin. Biol. Ther. 9, 427-440.

Ding, Z., Bivas-Benita, M., Hirschberg, H., Kersten, G.F., Jiskoot, W., Bouwstra, J.A., 2008. Preparation and characterization of diphtheria toxoid-loaded elastic vesicles for transcutaneous immunization. J. Drug Target. 16, 555-563.

Ding, Z., Van Riet, E., Romeijn, S., Kersten, G.F., Jiskoot, W., Bouwstra, J.A., 2009 Immune modulation by adjuvants combined with diphtheria toxoid administered topically in BALB/c mice after microneedle array pretreatment. Pharm. Res. 26, 1635-1643.

Ding, Z., Bal, S.M., Romeijn, S., Kersten, G.F., Jiskoot, W., Bouwstra, J.A., 2010. Transcutaneous immunization studies in mice using diphtheria toxoid-loaded vesicle formulations and a microneedle array. Pharm. Res. 28, 145-158.
El Maghraby, G.M., Williams, A.C., Barry, B.W., 1999. Skin delivery of oestradiol from deformable and traditional liposomes: mechanistic studies. J. Pharm. Pharmacol. 51, 1123-1134.

El Maghraby, G.M., Williams, A.C., Barry, B.W., 2000. Oestradiol skin delivery from ultradeformable liposomes: refinement of surfactant concentration. Int. J. Pharm. 196, 63-74.

Giudice, E.L., Campbell, J.D., 2006. Needle-free vaccine delivery. Adv. Drug Deliv. Rev., 68-89.

Glenn, G.M., Rao, M., Matyas, G.R., Alving, C.R., 1998. Skin immunization made possible by cholera toxin. Nature 391,851 .

Glenn, G.M., Flyer, D.C., Ellingsworth, L.R., Frech, S.A., Frerichs, D.M., Seid, R.C., Yu, J., 2007. Transcutaneous immunization with heat-labile enterotoxin: development of a needle-free vaccine patch. Expert Rev. Vaccines 6, 809-819.

Guebre-Xabier, M., Hammond, S.A., Epperson, D.E., Yu, J., Ellingsworth, L., Glenn, G.M., 2003. Immunostimulant patch containing heat-labile enterotoxin from Escherichia coli enhances immune responses to injected influenza virus vaccine through activation of skin dendritic cells 7. J. Virol. 77, 5218-5225.

Gupta, P.N., Mishra, V., Singh, P., Rawat, A., Dubey, P., Mahor, S., Vyas, S.P., 2005. Tetanus toxoid-loaded transfersomes for topical immunization. J. Pharm. Pharmacol. 57, 295-301.

Heurtault, B., Frisch, B., Pons, F., 2010. Liposomes as delivery systems for nasal vaccination: strategies and outcomes. Expert. Opin. Drug Deliv. 7, 829-844.

Hirschberg, H.J., van de Wijdeven, G.G., Kraan, H., Amorij, J.P., Kersten, G.F., 2010. Bioneedles as alternative delivery system for hepatitis B vaccine. J. Control Release 147, 211-217.

Isaka, M., Yasuda, Y., Mizokami, M., Kozuka, S., Taniguchi, T., Matano, K., Maeyama, J., Mizuno, K., Morokuma, K., Ohkuma, K., Goto, N., Tochikubo, K., 2001. Mucosal immunization against hepatitis B virus by intranasal co-administration of recombinant hepatitis $B$ surface antigen and recombinant cholera toxin B subunit as an adjuvant. Vaccine 19, 1460-1466.

Lebre, F., Borchard, G., de Lima, M.C., Borges, O., 2011. Progress towards a needlefree hepatitis B vaccine. Pharm. Res. 28, 986-1012.

Lowry, O.H., Rosebrough, N.J., Farr, A.L., Randall, R.J., 1951. Protein measurement with the Folin phenol reagent. J. Biol. Chem. 193, 265-275.

Mann, J.F., Acevedo, R., Campo, J.D., Perez, O., Ferro, V.A., 2009. Delivery systems: a vaccine strategy for overcoming mucosal tolerance? Expert Rev. Vaccines 8, 103-112.

Masheswari, C., Pandey, R.S., Chaurasiya, A., Kumar, A., Selvam, D.T., Prasad, G.B., Dixit, V.K., 2011. Non-ionic surfactant vesicles mediated transcutaneous immunization against Hepatitis B. Int. Immunopharmacol. 11, 1516-1522.

Mishra, D., Dubey, V., Asthana, A., Saraf, D.K., Jain, N.K., 2006. Elastic liposomes mediated transcutaneous immunization against Hepatitis B. Vaccine 24, 48474855 .

Oh, Y.K., Kim, M.Y., Shin, J.Y., Kim, T.W., Yun, M.O., Yang, S.J., Choi, S.S., Jung, W.W., Kim, J.A., Choi, H.G., 2006. Skin permeation of retinol in Tween 20-based deformable liposomes: in-vitro evaluation in human skin and keratinocyte models. J. Pharm. Pharmacol. 58, 161-166.

Paul, A., Cevc, G., Bachhawat, B.K., 1995. Transdermal immunization with large proteins by means of ultradeformable drug carriers 23. Eur. J. Immunol. 25, 3521-3524.

Paul, A., Cevc, G., Bachhawat, B.K., 1998. Transdermal immunisation with an integral membrane component, gap junction protein, by means of ultradeformable drug carriers, transfersomes. Vaccine 16, 188-195.

Prausnitz, M.R., Mikszta, J.A., Cormier, M., Andrianov, A.K., 2009. Microneedle-based vaccines. Curr. Top. Microbiol. Immunol. 333, 369-393.

Slutter, B., Bal, S., Keijzer, C., Mallants, R., Hagenaars, N., Que, I., Kaijzel, E., van Eden, W., Augustijns, P., Lowik, C., Bouwstra, J., Broere, F., Jiskoot, W., 2010. Nasal vaccination with $\mathrm{N}$-trimethyl chitosan and PLGA based nanoparticles: nanoparticle characteristics determine quality and strength of the antibody response in mice against the encapsulated antigen. Vaccine 28, 6282-6291.

Trotta, M., Peira, E., Carlotti, M.E., Gallarate, M., 2004. Deformable liposomes for dermal administration of methotrexate. Int. J. Pharm. 270, 119-125.

van den Bergh, B.A., Bouwstra, J.A., Junginger, H.E., Wertz, P.W., 1999. Elasticity of vesicles affects hairless mouse skin structure and permeability. J. Control Release 62, 367-379. 